mal trafficking profile (23). DEC-205, MMR, and DC-SIGN, but not langerin, contain the YXXФ motifs in cytoplasmic tails, suggesting that CD1 and C-type lectins share the same molecular sorting mechanisms for recycling to particular subcompartments (21).

In summary, each C-type lectin transports antigens to one or more specific subcompartments where selected antigen presentation molecules (i.e., MHC class I and II molecules and CD1 isoforms) recycle preferentially (Figure 1). Thus, C-type lectins govern not only the subcellular destination of newly captured antigens, but also their presentation pathway. In fact, geographic and functional coupling of $\mathrm{CD} 1 \mathrm{~b}$ and MMR has been reported to mediate CD1b-restricted presentation of mycobacterial lipoglycan antigens (24). The striking diversity in surface expression profiles of CD1 isoforms and C-type lectins among DC subsets and in intracellular trafficking routes of both classes of the receptors enables the DC system to survey a wide variety of tissues and subcompartments for carbohydrate-bearing antigens and to present distinct antigenic moieties to respective effector $\mathrm{T}$ cell populations.

Address correspondence to: Akira Takashima, Department of Dermatology, University of Texas Southwestern Medical Center, 5323 Harry Hines Boulevard, Dallas, Texas 75235, USA. Phone: (214) 648-3419; Fax: (214) 648-3472; E-mail: Akira.Takashima@ UTSouthwestern.edu.
1. Rowden, G., Lewis, M.G., and Sullivan, A.K. 1977. Ia antigen expression on human epidermal Langerhans cells. Nature. 268:247-248.

2. Klareskog, L., Tjernlund, U., Forsum, U., and Peterson, P.A. 1977. Epidermal Langerhans cells express Ia antigens. Nature. 268:248-250.

3. Wolff, K., and Winkelmann, R.K. 1967. Ultrastructural localization of nucleoside triphosphatase in Langerhans cells. J. Invest. Dermatol. 48:50-54.

4. Birbeck, M.S., Breathnach, A.S., and Everall, J.D. 1961. An electron microscope study of basal melanocytes and high-level clear cells (Langerhans cells) in vitiligo. J. Invest. Dermatol. 37:51-64.

5. Fithian, E., et al. 1981. Reactivity of Langerhans cells with hybridoma antibody. Proc. Natl. Acad. Sci. U. S. A. 78:2541-2544.

6. Tang, A., Amagai, M., Granger, L.G., Stanley, J.R., and Udey, M.C. 1993. Adhesion of epidermal Langerhans cells to keratinocytes mediated by E-cadherin. Nature. 361:82-85.

7. Valladeau, J., et al. 1999. The monoclonal antibody DCGM4 recognizes Langerin, a protein specific of Langerhans cells, and is rapidly internalized from the cell surface. Eur. J. Immunol. 29:2695-2704.

8. Mizumoto, N., et al. 2002. CD39 is the dominant Langerhans cell-associated ecto-NTPDase: modulatory roles in inflammation and immune responsiveness. Nat. Med. 8:358-365.

9. McDermott, R., et al. 2002. Birbeck granules are subdomains of endosomal recycling compartment in human epidermal Langerhans cells, which form where Langerin accumulates. Mol. Biol. Cell. 13:317-335.

10. Riedl, E., et al. 2000. Ligation of E-cadherin on in vitrogenerated immature Langerhans-type dendritic cells inhibits their maturation. Blood. 96:4276-4284.

11. Hunger, R.E., et al. 2004. Langerhans cells utilize CD1a and langerin to efficiently present nonpeptide antigens to T cells. J. Clin. Invest. 113:701-708. doi:10.1172/ JCI200419655.

12. Moody, D.B., and Porcelli, S.A. 2003. Intracellular pathways of CD1 antigen presentation. Nat. Rev. Immunol. 3:11-22.

13. Sugita, M., et al. 1999. Separate pathways for antigen presentation by CD1 molecules. Immunity. 11:743-752.
14. Pena-Cruz, V., Ito, S., Dascher, C.C., Brenner, M.B., and Sugita, M. 2003. Epidermal Langerhans cells efficiently mediate CD1a-dependent presentation of microbial lipid antigens to T cells. J. Invest. Dermatol. 121:517-521.

15. Salamero, J., et al. 2001. CD1a molecules traffic through the early recycling endosomal pathway in human Langerhans cells. J. Invest. Dermatol. 116:401-408.

16. Valladeau, J., et al. 2000. Langerin, a novel C-type lectin specific to Langerhans cells, is an endocytic receptor that induces the formation of Birbeck granules. Immunity. 12:71-81.

17. Jiang, W., et al. 1995. The receptor DEC-205 expressed by dendritic cells and thymic epithelial cells is involved in antigen processing. Nature. 375:151-155.

18. Sallusto, F., Cella, M., Danieli, C., and Lanzavecchia, A. 1995. Dendritic cells use macropinocytosis and the mannose receptor to concentrate macromolecules in the major histocompatibility complex class II compartment: Downregulation by cytokines and bacterial products. J. Exp. Med. 182:389-400.

19. Geijtenbeek, T.B., et al. 2000. Identification of DC-SIGN, a novel dendritic cell-specific ICAM-3 receptor that supports primary immune responses. Cell. 100:575-585.

20. Turville, S.G., et al. 2002. Diversity of receptors binding HIV on dendritic cell subsets. Nat. Immunol. 3:975-983.

21. Figdor, C.G., van Kooyk, Y., and Adema, G.J. 2002. $C$-type lectin receptors on dendritic cells and Langerhans cells. Nat. Rev. Immunol. 2:77-84.

22. Mahnke, K., et al. 2000. The dendritic cell receptor for endocytosis, DEC-205, can recycle and enhance antigen presentation via major histocompatibility complex class II-positive lysosomal compartments. J. Cell Biol. 151:673-684.

23. Engering, A., et al. 2002. The dendritic cell-specific adhesion receptor DC-SIGN internalizes antigen for presentation to T cells. J. Immunol. 168:2118-2126.

24. Prigozy, T.I., et al. 1997. The mannose receptor delivers lipoglycan antigens to endosomes for presentation to $\mathrm{T}$ cells by $\mathrm{CD} 1 \mathrm{~b}$ molecules. Immunity. 6:187-197.

\title{
Viral immunosuppression: disabling the guards
}

\author{
Marco Colonna
}

Department of Pathology and Immunology, Washington University School of Medicine, St. Louis, Missouri, USA.

\begin{abstract}
When facing an immune response, viruses can either attempt to elude them or confront them. A new report demonstrates that a lymphocytic choriomeningitis virus (LCMV) strain can suppress immune responses by targeting both development and activation of DCs (see the related article beginning on page 737). Ironically, type I IFN released in response to LCMV infection contributes to the blockade of DC development. The discovery of these immunosuppressive mechanisms provides new perspectives for the therapy of chronic infections associated with immunosuppression.
\end{abstract}

Nonstandard abbreviations used: $\alpha$-dystroglycan $(\alpha-\mathrm{DG})$; Armstrong 53b (ARM); clone 13 (Cl 13); natural IFN-producing cell (IPC); lymphocytic choriomeningitis virus (LCMV).

Conflict of interest: The author has declared that no conflict of interest exists.

Citation for this article:

J. Clin. Invest. 113:660-662 (2004).

doi:10.1172/JCI200421166.
Viruses have evolved multiple strategies to counteract host immune responses. Noncytopathic lymphocytic choriomeningitis virus (LCMV) employs several of these strategies to successfully infect mice. Initial immunosurveillance of LCMV infection is mediated by CTLs. However, this response may lead to selection of LCMV variants that carry mutations in the relevant CTL epitopes and, therefore, can elude cytotoxic responses $(1,2)$. Antibody responses are also essential for long-term protection. However, LCMV variants can evade humoral responses with point mutations that encode novel amino acids distorting the envelope glycoprotein epitope recognized by neutralizing antibodies (3).

\section{DCs as targets of LCMV immunosuppression}

Remarkably, LCMV not only eludes specific immune surveillance, but can also actively suppress immune responses. How is this accomplished? In this issue of the JCI, Sevilla and colleagues elucidate the mechanism 


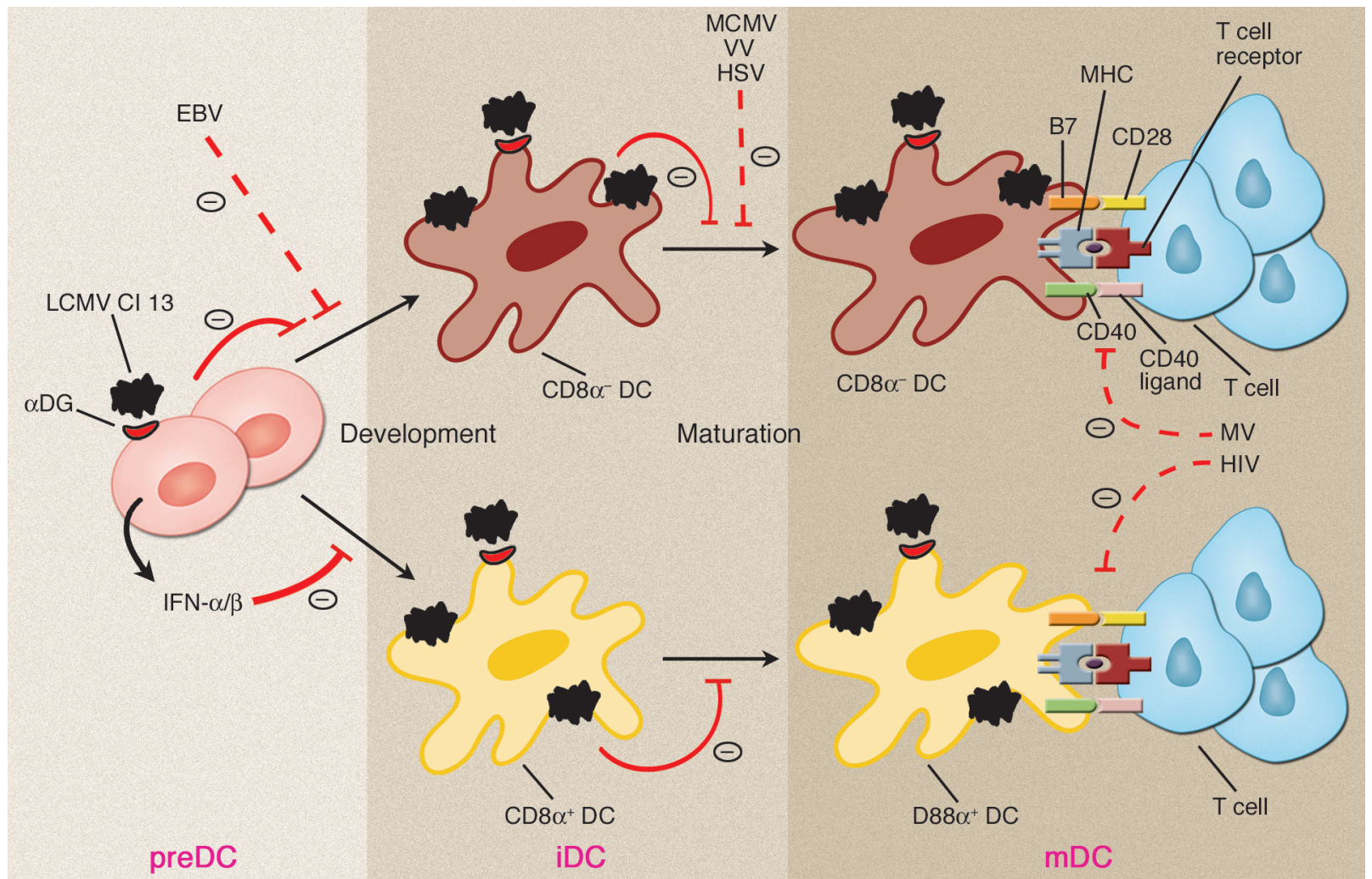

Figure 1

Immunosuppressive mechanisms of LCMV Cl 13. Cl 13 infects DC precursors and DCs, possibly using $\alpha$-DG as the entry receptor. Cl 13 blocks development of CD8 $\alpha^{+}$and CD8 $\alpha^{-}$DCs from DC precursors (preDCs) and prevents immature DCs (iDCs) from becoming mature DCs (mDCs), which express high levels of MHC, CD40, and B7, and initiate T cell responses. Blockade of CD8 $\alpha^{+}$DC development by Cl 13 requires type I IFN. The sites of action of other immunosuppressive viruses interfering with DC functions or DC-T cell interactions are indicated. EBV, Epstein-Barr virus; HSV, herpes simplex virus; VV, vaccinia virus; MCMV, murine cytomegalovirus; MV, measles virus.

used by an immunosuppressive LCMV variant known as LCMV clone 13 (Cl 13) (4). This viral variant was originally isolated from the lymphoid tissues of neonatal mice with a persistent LCMV infection induced by the nonimmunosuppressive wild-type LCMV, Armstrong 53b (ARM) (5). Initial studies indicated that $\mathrm{Cl} 13$ targets $\mathrm{APCs}$, and, in particular DCs (6). DCs are sentinels of the immune system; they efficiently capture viral antigens at the infection site and rapidly migrate to the lymph nodes where they initiate $\mathrm{T}$ cell responses (7). Thus, DCs are ideal targets for viral immunosuppression. Sevilla and colleagues demonstrate that $\mathrm{Cl} 13$ adopts a surprising dual strategy for disabling DCs: inhibiting both their development and $\mathrm{T}$ cell stimulatory function (Figure 1). They clearly show that infection with $\mathrm{Cl}$ 13 impairs the expression of $\mathrm{MHC}$ and costimulatory molecules on both spleen myeloid $\left(\mathrm{CD} 8 \alpha^{-}\right)$and lymphoid $\left(\mathrm{CD} 8 \alpha^{+}\right)$ DCs. As a result, DCs do not efficiently stimulate $\mathrm{T}$ cell proliferation ex vivo. DC function remains impaired as long as LCMV infection persists. Moreover, Cl 13 infects bone marrow precursors in vivo and in vitro, inhibiting development and differentiation of CD8 $\alpha^{-}$and CD8 $\alpha^{+}$DCs.

Why does Cl 13 selectively affect DCs and their precursors? LCMV has been shown to bind $\alpha$-dystroglycan $(\alpha-D G)$, a receptor for extracellular matrix proteins that is highly expressed on DCs and bone marrow precursors (8). Notably, $\mathrm{Cl} 13$ has a higher affinity for $\alpha$-DG than does wild-type ARM (8). Thus, it is possible that $\mathrm{Cl} 13$ competes with extracellular matrix proteins for binding $\alpha$-DG on DCs, thereby infecting them, whereas ARM does not.

\section{The paradoxical role of type I IFN}

The notion that DCs are major targets of immunosuppressive viruses is corroborated by several other types of infections (Figure 1$)$. Measles virus $(9,10)$, herpes simplex virus (11), vaccinia virus (12), and murine cytomegalovirus (13) infect DCs and impair their capacity to stimulate T cells. Human immunodeficiency virus exploits
DCs for transmission to $\mathrm{T}$ cells (14). Epstein-Barr virus inhibits the development of DCs by inducing apoptosis of their monocytic precursors without infecting them (15). Furthermore, DCs are eliminated by CTL-mediated responses elicited by some immunosuppressive LCMV variants (16). In comparison with these immunosuppressive mechanisms, the inhibition of DCs by $\mathrm{Cl} 13$ reported by Sevilla and colleagues is remarkable in that the virus impairs both DC immunostimulatory function and the development of CD8 $\alpha^{-}$ and CD $8 \alpha^{+}$DCs. Moreover, Sevilla and colleagues demonstrate that type I IFN, i.e., IFN- $\alpha$ and/or IFN- $\beta$, is necessary for $\mathrm{Cl}$ 13 -mediated blockade of CD8 $\alpha^{+}$DC development. Thus, type I IFN paradoxically contributes to immunosuppression rather than host defense. What is the mechanism? Previous studies have shown that $\mathrm{Cl} 13$, in contrast to wild-type ARM, effectively triggers secretion of type I IFN by DCs (17). Thus, type I IFN may affect CD $8 \alpha^{+}$DC development through an autocrine loop. 
The paracrine action of type I IFN secreted by natural IFN-producing cells (IPCs) (18) in response to $\mathrm{Cl} 13$ may also be involved. Another intriguing observation reported by Sevilla and colleagues is that $\mathrm{Cl} 13$ inhibits expression of MHC in DCs cultured from bone marrow cells, although type I IFN would be expected to increase at least MHC class I expression. Whether $\mathrm{Cl} 13$ directly inhibits MHC synthesis by mechanisms similar to those employed by herpes viruses (19) remains to be determined.

In conclusion, the $\mathrm{Cl} 13$ infection model underscores the central role of DCs in mediating viral immunosuppression and describes novel methods by which a virus can impair DCs. It will be important to investigate the influence of viral burden on these mechanisms. Infection of bone marrow and suppression of DC development may require higher viral loads than infection of peripheral DCs. Another important question is whether Cl 13 immunosuppression involves other APCs that may participate in anti-LCMV immune responses, such as IPCs and macrophages. Certainly, $\mathrm{Cl} 13$ infection will provide a valuable model to test whether increasing DC numbers, their maturation, and their $T$ cell stimulatory capacity can improve the efficacy of vaccines in chronic infections associated with immunosuppression.
Address correspondence to: Marco Colonna, Department of Pathology and Immunology, Washington University School of Medicine, Box 8118, 660 South Euclid Avenue, St. Louis, Missouri 63110, USA. Phone: (314) 362-0367; Fax: (314) 362-4096; E-mail: mcolonna@pathology.wustl.edu.

1. Oldstone, M.B. 1997. How viruses escape from cytotoxic T lymphocytes: molecular parameters and players. Virology. 234:179-185.

2. Klenerman, P., and Zinkernagel, R.M. 1998. Original antigenic sin impairs cytotoxic $\mathrm{T}$ lymphocyte responses to viruses bearing variant epitopes. Nature. 394:482-485.

3. Ciurea, A., et al. 2000. Viral persistence in vivo through selection of neutralizing antibody-escape variants. Proc. Natl. Acad. Sci. U. S. A. 97:2749-2754.

4. Sevilla, N., McGavern, D.B., Teng, C., Kunz, S., and Oldstone, M.B.A. 2004. Viral targeting of hematopoietic progenitors and inhibition of DC maturation as a dual strategy for immune subversion. J. Clin. Invest. 113:737-745. doi:10.1172/JCI200420243.

5. Ahmed, R., and Oldstone, M.B. 1988. Organ-specific selection of viral variants during chronic infection. J. Exp. Med. 167:1719-1724.

6. Sevilla, N., et al. 2000. Immunosuppression and resultant viral persistence by specific viral targeting of dendritic cells. J. Exp. Med. 192:1249-1260.

7. Banchereau, J., and Steinman, R.M. 1998. Dendritic cells and the control of immunity. Nature. 392:245-252.

8. Sevilla, N., Kunz, S., McGavern, D., and Oldstone, M.B. 2003. Infection of dendritic cells by lymphocytic choriomeningitis virus. Curr. Top. Microbiol. Immunol. 276:125-144.

9. Servet-Delprat, C., Vidalain, P.O., Valentin, H., and Rabourdin-Combe, C. 2003. Measles virus and den- dritic cell functions: how specific response cohabits with immunosuppression. Curr. Top. Microbiol. Immunol. 276:103-123.

10. Karp, C.L., et al. 1996. Mechanism of suppression of cell-mediated immunity by measles virus. Science. 273:228-231.

11. Salio, M., Cella, M., Suter, M., and Lanzavecchia, A. 1999. Inhibition of dendritic cell maturation by herpes simplex virus. Eur. J. Immunol. 29:3245-3253.

12. Engelmayer, J., et al. 1999. Vaccinia virus inhibits the maturation of human dendritic cells: a novel mechanism of immune evasion. J. Immunol. 163:6762-6768.

13. Andrews, D.M., Andoniou, C.E., Granucci, F., Ricciardi-Castagnoli, P., and Degli-Esposti, M.A. 2001. Infection of dendritic cells by murine cytomegalovirus induces functional paralysis. Nat. Immunol. 2:1077-1084.

14. Steinman, R.M., et al. 2003. The interaction of immunodeficiency viruses with dendritic cells. Curr. Top. Microbiol. Immunol. 276:1-30.

15. Li, L., et al. 2002. Epstein-Barr virus inhibits the development of dendritic cells by promoting apoptosis of their monocyte precursors in the presence of granulocyte macrophage-colony-stimulating factor and interleukin-4. Blood. 99:3725-3734.

16. Odermatt, B., Eppler, M., Leist, T.P., Hengartner, H., and Zinkernagel, R.M. 1991. Virus-triggered acquired immunodeficiency by cytotoxic T-cell-dependent destruction of antigen-presenting cells and lymph follicle structure. Proc. Natl. Acad. Sci. U. S. A. 88:8252-8256.

17. Diebold, S.S., et al. 2003. Viral infection switches nonplasmacytoid dendritic cells into high interferon producers. Nature. 424:324-328.

18. Colonna, M., Krug, A., and Cella, M. 2002. Interferonproducing cells: on the front line in immune responses against pathogens. Curr. Opin. Immunol. 14:373-379.

19. Tortorella, D., Gewurz, B.E., Furman, M.H., Schust, D.J., and Ploegh, H.L. 2000. Viral subversion of the immune system. Annu. Rev. Immunol.18:861-926.

\title{
The anatomy of an arrhythmia
}

\author{
Robert F. Gilmour, Jr.
}

\author{
Department of Biomedical Sciences, Cornell University, Ithaca, New York, USA.
}

\begin{abstract}
Computer simulations are potentially effective approaches to unraveling the causes of lethal heart rhythm disorders. In this issue of the JCI, Xie et al. (see the related article beginning on page 686) have embedded a well-characterized dynamic mechanism for arrhythmia development in an anatomically realistic computer model of the heart. Their demonstration that this simple mechanism governs the behavior of the complex model may provide a new target for strategies to prevent sudden death.
\end{abstract}

\section{Ventricular fibrillation and sudden cardiac death}

The primary mechanical event in the heart-the development of contractile force-is triggered by an electrical event, the

Nonstandard abbreviations used: action potential duration (APD); atrioventricular (AV); conduction velocity (CV); sinoatrial (SA); ventricular fibrillation (VF). Conflict of interest: The author has declared that no conflict of interest exists.

Citation for this article:

J. Clin. Invest. 113:662-664 (2004).

doi:10.1172/JCI200421223. cardiac action potential, through the process of excitation-contraction coupling. For the heart to contract efficiently and continuously over the life span of an individual, which may encompass many millions of heartbeats, electrical activation of the heart must occur repetitively in the proper sequence. Orderly electrical activation is accomplished by the sequential propagation of action potentials along the anatomically defined structures shown in the right panel of Figure 1. The heartbeat begins in the sinoatrial (SA) node with a sponta- neously generated action potential. Propagation of the SA nodal impulse creates wavefronts of electrical excitation that initially spread outward to atrial myocardium and then converge before crossing the atrioventricular (AV) node and entering the specialized conducting system, which consists of the bundle branches and an arborizing network of Purkinje cells. The Purkinje system then distributes activation rapidly and widely to ventricular myocardium.

If the sequence of electrical activation becomes disorganized, the mechanical activity of the heart is compromised. In the most extreme case of disorganization, ventricular fibrillation (VF), the electrical activity of the ventricles becomes so rapid and irregular that coordinated contraction ceases, causing blood pressure to plummet and death to ensue within minutes. Despite decades of intensive investigation, sudden death from 